\title{
Multiple Sclerosis Journal
}

http://msj.sagepub.com/

Can the functional assessment of multiple sclerosis adapt to changing needs? A psychometric validation in patients with clinically isolated syndrome and early relapsing -remitting multiple sclerosis

S Acaster, P Swinburn, C Wang, B Stemper, K Beckmann, V Knappertz, C Pohl, R Sandbrink, K Gondek, G Edan, L Kappos, M Freedman, H-P Hartung, B Arnason, G Comi, M Filippi, D Jeffery, P O'Connor, S Cook and AJ Lloyd Mult Scler 2011 17: 1504 originally published online 14 July 2011

DOI: $10.1177 / 1352458511414039$

The online version of this article can be found at:

http://msj.sagepub.com/content/17/12/1504

\author{
Published by: \\ (3)SAGE \\ http://www.sagepublications.com
}

Additional services and information for Multiple Sclerosis Journal can be found at:

Email Alerts: http://msj.sagepub.com/cgi/alerts

Subscriptions: http://msj.sagepub.com/subscriptions

Reprints: http://www.sagepub.com/journalsReprints.nav

Permissions: http://www.sagepub.com/journalsPermissions.nav

>> Version of Record - Nov 30, 2011

OnlineFirst Version of Record - Jul 14, 2011

What is This? 


\title{
Can the functional assessment of multiple sclerosis adapt to changing needs? A psychometric validation in patients with clinically isolated syndrome and early relapsing-remitting multiple sclerosis
}

Multiple Sclerosis Journal

17(12) 1504-1513

(c) The Author(s) 2011

Reprints and permissions:

sagepub.co.uk/journalsPermissions.nav DOI: 10.1 I77//3524585 I/414039 msj.sagepub.com

@SAGE

\author{
S Acaster', P Swinburn', C Wang', B Stemper ${ }^{3,4}$, \\ K Beckmann ${ }^{3}$, V Knappertz ${ }^{2}$, C Pohl ${ }^{3,5}$, R Sandbrink ${ }^{3,6}$, \\ K Gondek ${ }^{2}$, G Edan', L Kappos ${ }^{8}$, M Freedman', H-P Hartung ${ }^{6}$, \\ B Arnason ${ }^{10}$, G Comi ${ }^{11}$, M Filippi ${ }^{12}$, D Jeffery ${ }^{13}$, P O'Connor ${ }^{14}$, \\ S Cook' ${ }^{15}$ and AJ Lloyd'
}

\begin{abstract}
Background: The Functional Assessment of Multiple Sclerosis (FAMS) is widely used in clinical trial programmes; however, it was developed before the rise in trials targeted at early stage multiple sclerosis (MS) and clinically isolated syndrome (CIS).

Objective: The aim of this study was to assess the psychometric properties of the FAMS within two clinically distinct populations, CIS and early relapsing-remitting MS (RRMS), and discern the appropriateness of the FAMS within these populations.

Methods: Secondary analysis was conducted on FAMS data from two clinical trials assessing interferon beta-Ib in early RRMS and CIS. The statistical analysis assessed the scale acceptability, reliability, validity and responsiveness of the FAMS. Item response theory (IRT) was also conducted on the early RRMS sample in order to assess how well the FAMS discriminated amongst individuals with less severe MS.

Results: Results from both trials demonstrated an improvement in the FAMS psychometric properties with increased baseline disease severity. However, high ceiling effects were evident amongst less severe patients, and there was an overall lack of responsiveness to improvement and poor construct validity. IRT also demonstrated its lack of discrimination/sensitivity in early RRMS.

Conclusions: In trials involving patients with early stage RRMS and CIS, modifications to the FAMS based on a qualitative assessment of its content validity in these populations would be required in order to potentially improve the FAMS psychometric properties and sensitivity.
\end{abstract}

\footnotetext{
'Oxford Outcomes Ltd, Oxford, UK.

${ }^{2}$ Bayer HealthCare Pharmaceuticals, Montville, NJ, USA.

${ }^{3}$ Bayer Schering Pharma, Berlin, Germany.

${ }^{4}$ Department of Neurology, University of Erlangen-Nurnberg, Germany.

${ }^{5}$ Medical University Clinic, Bonn, Germany.

${ }^{6}$ Heinrich-Heine-Universität, Düsseldorf, Germany.

${ }^{7}$ Centre Hospitalier Universitaire, Rennes, France.

${ }^{8}$ University Hospital, Basel, Switzerland.

${ }^{9}$ Ottawa Hospital - General Campus, Ottawa, Canada.

${ }^{10}$ Surgery Brain Research Institutes, Chicago, IL, USA.
}

\footnotetext{
"Department of Neurology and Clinical Neurophysiology, Vita Salute University, Milan, Italy.

${ }^{12}$ Neuroimaging Research Unit, Scientific Institute and University, Ospedale San Raffaele, Milan, Italy.

${ }^{13}$ Department of Neurology, Wake Forest University Baptist Medical Centre, Winston-Salem, NC, USA.

${ }^{14}$ St Michael's Hospital, Toronto, Ontario, Canada.

${ }^{15}$ UMD New Jersey Medical School, Newark, NJ, USA.

Corresponding author:

Sarah Acaster, Oxford Outcomes Ltd, Seacourt Tower, West Way, Oxford, OX2 0JJ, UK

Email: sarah.acaster@oxfordoutcomes.com
} 


\section{Keywords}

clinically isolated syndrome (CIS), Functional Assessment of Multiple Sclerosis (FAMS), multiple sclerosis, psychometric validation

Date received: 24th January 20I I; revised: I2th May 20II; accepted: I8th May 20II

\section{Introduction}

Patient reported outcome (PRO) assessments of healthrelated quality of life (HRQL) are increasingly incorporated into clinical trials. ${ }^{1}$ A number of generic and disease specific HRQL measures are widely accepted for use in the multiple sclerosis (MS) population, ${ }^{2}$ and there is evidence that such measures are more capable of capturing the overall impact of MS compared with some clinical or physical disability scales, such as the Expanded Disability Status Scale (EDSS). ${ }^{3,4}$ Given the increased use of HRQL measures, and the potential for PRO-related labelling or promotional claims in MS, it is imperative that measures used in clinical trials are appropriate from both conceptual and psychometric perspectives.

One of the most widely used PRO measures in MS is the Functional Assessment of Multiple Sclerosis (FAMS). ${ }^{5}$ However the FAMS was developed before the rise in trials targeted at early-stage MS and suspected prodromal MS. Thus, whilst the psychometric properties of the FAMS within an MS population have been demonstrated, ${ }^{5-8}$ all validation studies to date have involved patients with moderate to severe MS (mean EDSS ratings 3.5-6.4). There has been no investigation of the psychometric properties of the FAMS in an early MS population or in people with clinically isolated syndrome (CIS). The latter is unsurprising given that a clear official definition of the term CIS was only established after the development of the FAMS; as described in the European Medicines Agency (EMA) draft and final guidelines on the treatment of MS. ${ }^{9,10}$ Given increasing clinical research in the field of treatments for early stage MS and patients with CIS, it is essential that the appropriateness of the FAMS is established if it is to be used in these trials.

This report details secondary analysis of FAMS data from two clinical trials assessing interferon beta $-1 b$ (IFN $\beta-1 b$ ) in two distinct populations: one comprising a baseline CIS sample (BENEFIT) ${ }^{11-13}$ and one with an early MS sample (BEYOND). ${ }^{14}$ The aim of this analysis was to assess the psychometric properties of the FAMS in previously unrepresented populations. In addition to conventional psychometric methods, item response theory (IRT) was also conducted on the early MS population sample. IRT analysis is increasingly being applied to PRO questionnaire development, evaluation and refinement, ${ }^{15}$ and has been integral to the development of new PRO databanks such as the PROMIS project. ${ }^{16}$ The application of IRT analysis in the present study was conducted in order to assess how well the FAMS was able to discriminate amongst individuals with less severe MS. This represents a novel approach to the analysis of MS PRO data, because whilst the use of IRT on the FAMS has been recommended, it has not yet been reported, to our knowledge. $^{8}$

\section{Materials and methods}

\section{Samples}

The BENEFIT study assessed early versus delayed treatment with IFN $\beta-1 b$ in patients with CIS and a minimum of two clinically silent lesions on brain MRI. The initial study was a double-blind, placebocontrolled, randomized control trial; the study duration was until first relapse or, at maximum, 2 years. The 3 -year follow-up study included $89 \%$ of the original sample, was open-label, and offered active treatment to all those previously in the placebo group. The valid baseline FAMS sample included 349 adult patients, of whom 340 patients provided valid end of study (5-year follow-up) or 'last observation carried forward' FAMS data.

The BEYOND study compared IFN $\beta-1 b$ dosages $(250 \mu \mathrm{g}$ vs. $500 \mu \mathrm{g})$ in treatment naïve patients with early relapsing-remitting MS (RRMS). It was a double-blind, prospective, multicentre, randomized trial; the study duration was at least 2 years, continuing until the last patient had completed 2 years. At baseline the BEYOND sample included 1644 adult patients from the IFN $\beta-1 b$ treatment arms who provided valid FAMS data, and of these 1590 patients provided valid end of study or last observation carried forward FAMS data.

Full descriptions of the BENEFIT and BEYOND trial designs and samples are provided elsewhere. ${ }^{11-14}$

\section{Measures}

The following three measures were utilized in the analysis: the FAMS, the EDSS, and the Multiple Sclerosis 
Functional Composite (MSFC). ${ }^{17}$ The FAMS is a selfreported multidimensional index of HRQL comprising a total of 58 items on the following subscales: mobility (7 items), symptoms (7 items), emotional well-being (7 items), general contentment (7 items), thinking and fatigue (9 items), family/social well-being ( 7 items), and additional concerns (14 items). Each item is rated on a 5-point scale (0-4); after reverse scoring negatively worded items, a higher score reflects a higher quality of life. Each domain can be scored and a total score can be derived by summing all domains except 'additional concerns', giving a possible range of $0-176$. A trial outcome index score (TOI; $0-148$ ) can also be derived by summing the mobility, symptoms, thinking and fatigue, and additional concerns domains.

The EDSS is a physician-completed rating system for classifying the extent of MS related impairment, which ranges from 0 (normal neurological exam) to 10 (death due to MS). The MSFC is a clinical test battery devised for use in MS trials and consists of three functional tests: a timed 25 -foot $(7.6 \mathrm{~m})$ walk (ambulation), the 9-hole peg test (arm function) and the paced auditory serial addition tests (PASAT; cognitive function). The ambulation and arm function tests are measured in seconds and the PASAT is scored by the number of correct responses. Each test score can be standardized and the sum of these standardized scores provides the MSFC total score.

The EDSS ratings were used to stratify each of the samples, and relevant MSFC data were used to assess concurrent validity in the FAMS mobility and thinking and fatigue domains, and the total and TOI scores.

\section{Statistical analysis}

The psychometric properties of the FAMS were evaluated using four standard statistical assessments in each of the samples: data acceptability, internal consistency reliability, construct validity, and responsiveness. Data acceptability was determined by examining score distributions; acceptability is supported when observed scores are well distributed, mean scores are near the scale mid-point, and floor and ceiling effects are below $15 \% .{ }^{18}$ Internal consistency reliability was determined using Cronbach's alpha coefficient; an alpha value of $0.70-0.90$ was used as a guideline for determining that a subscale or score was internally consistent, ${ }^{19}$ without being overly homogenous. ${ }^{20}$ Construct validity assesses the extent to which an instrument measures or correlates with the concept it purports to measure. The total and TOI FAMS scores validity was determined by examining the extent to which they correlated with the MSFC total scores; the validity of the Mobility and 'Thinking and fatigue' domains were determined by examining the correlations with the MSFC ambulation and cognition tests, respectively. Responsiveness was examined by comparing baseline and end of study or last observation carried forward FAMS scores, by EDSS progression groups using paired sample $t$-test, and standardized response means. Three groups were created based on input from clinicians: worsened (EDSS increased by $>0.5$ from baseline), stable (no EDSS change or \pm 0.5 from baseline) and improved (EDSS decreased by $>0.5$ from baseline). The standardized response mean (SRM) is an effect size measurement which reflects the ratio of the mean score change to the standard deviation (SD) of the score change. There are no definitive standards for the SRM, but it has been suggested that Cohen's standard effect size model applies, with small, moderate and large responsiveness corresponding with SRMs of $0.20-0.49,0.50-0.79$ and 0.80 or more, respectively. ${ }^{21,22}$

In all instances the analyses were conducted on each trial sample as a whole and also on each trial sample split into two baseline EDSS groups: $0-1.5$ and $2+$. This split indicates the point at which the EDSS rating includes minimal disability and thus permits further investigation of the validity of the FAMS within the early MS population.

In addition to the above, the BEYOND data set was also investigated using item response theory (IRT), which was conducted in order to examine the extent to which each domain was able to discriminate amongst high functioning (low severity) patients with MS. Prior to analysis each domain was assessed to ensure it complied with the IRT uni-dimensionality assumption, using exploratory factor analysis (EFA). Given the ordinal response options in the FAMS, each domain was then calibrated using a graded response model. ${ }^{23}$

Calibration refers to the process of creating a metric for the latent trait of interest (e.g. Mobility); the responses to all items in the domain of interest are calibrated to create a standardized metric (commonly depicted as $\theta$ ) with a mean of zero and standard deviation of 1 . The value of $\theta$ (plotted on the $x$-axis) reflects the value of the latent trait (e.g. Mobility). Test information curves were generated for each domain in order to visually assess the level of discrimination/reliability in the FAMS domains amongst high functioning individuals. Higher information denotes more precision (reliability) for measuring a person's trait level (e.g. Mobility). Thus, the test information curves illustrate whether a domain is reliably measuring $\theta$ (or the FAMS domain of interest) at the less severe end of the spectrum.

All of the standard psychometric analyses were conducted using SPSS; version 17. The IRT analysis was conducted using M Plus; version 5.21. 
Table I. Sample characteristics

\begin{tabular}{|c|c|c|c|c|c|c|}
\hline \multirow[b]{2}{*}{ Characteristics } & \multicolumn{3}{|l|}{ BENEFIT } & \multicolumn{3}{|l|}{ BEYOND } \\
\hline & $\begin{array}{l}\text { Overall } \\
(n=348)\end{array}$ & $\begin{array}{l}\text { EDSS 0-I.5 } \\
(n=192)\end{array}$ & $\begin{array}{l}\text { EDSS } 2+ \\
(n=156)\end{array}$ & $\begin{array}{l}\text { Overall } \\
(n=1644)\end{array}$ & $\begin{array}{l}\text { EDSS } 0-1.5 \\
(n=537)\end{array}$ & $\begin{array}{l}\text { EDSS } 2+ \\
(n=110 \mathrm{I})\end{array}$ \\
\hline Age, mean (SD) & 30.91 (7.44) & $30.47(7.31)$ & $31.46(7.59)$ & $35.88(9.25)$ & 33.51 (8.69) & $37.06(9.27)$ \\
\hline Age range & $18-45$ & $18-45$ & $18-45$ & $18-57$ & $18-55$ & $18-57$ \\
\hline Female \% & 69.1 & 69.4 & 68.6 & 69.8 & 70.8 & 69.4 \\
\hline Caucasian \% & - & - & - & 91.0 & 92.6 & 90.3 \\
\hline Baseline EDSS, median (IQR) & $1.5(1.0-2.0)$ & I (I.0-I.5) & $2(2.0-2.5)$ & $2(1.5-3.0)$ & I.5 (I.0-I.5) & $3(2.0-3.5)$ \\
\hline Baseline EDSS range & $0-4.0$ & $0-1.5$ & $2-4.0$ & $0-6.5$ & $0-1.5$ & $2-6.5$ \\
\hline Progressed to CDMS \% & 47.9 & 45.1 & 51.3 & - & - & - \\
\hline Age at onset, mean (SD) & - & - & - & $31.14(9.18)$ & $30.14(8.53)$ & $31.64(9.45)$ \\
\hline Age at onset, range & - & - & - & $9-55$ & $11-55$ & $9-55$ \\
\hline Disease duration (years), mean (SD) & - & - & - & $5.30(5.80)$ & $3.94(4.55)$ & $5.98(6.22)$ \\
\hline Disease duration (years), range & - & - & - & $0-42$ & $0-32$ & $0-42$ \\
\hline \multicolumn{7}{|l|}{ Treatment } \\
\hline IFNB-Ib $250 \mu \mathrm{g} /$ placebo \% & $63.0 / 37.0$ & $61.7 / 38.3$ & $64.7 / 35.3$ & - & - & - \\
\hline IFNB-Ib $250 \mu g / 500 \mu g \%$ & - & - & - & $50.0 / 50.0$ & $49.0 / 51.0$ & $50.5 / 49.5$ \\
\hline
\end{tabular}

$\mathrm{IQR}=$ inter quartile range; IFN $\beta-\mathrm{Ib}=$ interferon beta- $\mathrm{Ib}$.

\section{Results}

\section{Sample characteristics}

Table 1 shows the characteristics of patients from the BENEFIT and BEYOND samples. Those in the BEYOND sample are older, and have a slightly higher EDSS median and range than those in the BENEFIT sample.

\section{Data acceptability}

Data from BENEFIT were skewed towards better quality of life; no patients reported the most extreme impairment on any domain (Table 2). In the EDSS 0-1.5 group, all domains had ceiling effects which exceeded the recommended maximum of $15 \%$ with the exception of 'general contentment' and 'additional concerns'. The EDSS 2+ group exceeded the recommended maximum ceiling effect in mobility, symptoms and emotional well-being. In both groups the level of missing data associated with each domain was negligible or none existent.

The BEYOND data were also skewed towards better quality of life. In the EDSS 0-1.5 group, four domains had ceiling effects which exceeded the recommended maximum: mobility, symptoms, emotional well-being and family/social well-being. However, in the EDSS $2+$ group, four domains spanned the entire scale range (symptoms, emotional well-being, thinking and fatigue, and family/social well-being), and no ceiling effects exceeded the recommended maximum. In both groups the level of missing data associated with each domain was negligible or none existent, with the highest reported level of $1.2 \%$ for the 'additional concerns' domain.

\section{Internal consistency reliability}

Internal consistency estimates were mostly found to be acceptable (Table 2). The exceptions were: 'thinking and fatigue' in the BENEFIT EDSS $2+$ sample which exceeded 0.9, and 'mobility' in the BEYOND EDSS $0-1.5$ group, which was just below 0.7 .

\section{Construct validity}

There was little evidence of construct validity within the BENEFIT sample, with the highest correlation $<0.4$ (Table 3). The pattern of results suggests improved validity with increased MS severity. However, the FAMS mobility and MSFC arm function correlation represents an anomaly, as the association between the FAMS score and MSFC assessment is greater amongst the less severe patients.

The BEYOND results demonstrated some evidence of construct validity, with two correlations exceeding 0.4. There was also a clearer pattern of improved construct validity with MS severity.

\section{Responsiveness}

Amongst the worsened group the BENEFIT EDSS 0-1.5 sub group mostly showed small effect sizes, but demonstrated moderate responsiveness on the 
Table 2. Functional Assessment of Multiple Sclerosis Scale acceptability and internal consistency reliability: baseline data

\begin{tabular}{|c|c|c|c|c|c|c|c|c|c|c|}
\hline \multirow[b]{2}{*}{ FAMS domain } & \multicolumn{5}{|l|}{ BENEFIT } & \multicolumn{5}{|c|}{ BEYOND } \\
\hline & $\begin{array}{l}\text { Score range } \\
\text { (Scale range) }\end{array}$ & $\begin{array}{l}\text { Mean } \\
\text { score (SD) }\end{array}$ & $\begin{array}{l}\text { Floor/ceiling } \\
\%\end{array}$ & $\begin{array}{l}\text { Cronbach's } \\
\text { alpha }\end{array}$ & $\begin{array}{l}\text { Missing } \\
\text { data }(n)\end{array}$ & $\begin{array}{l}\text { Score } \\
\text { range }\end{array}$ & $\begin{array}{l}\text { Mean } \\
\text { score (SD) }\end{array}$ & $\begin{array}{l}\text { Floor/ceiling } \\
\%\end{array}$ & $\begin{array}{l}\text { Cronbach's } \\
\text { alpha }\end{array}$ & $\begin{array}{l}\text { Missing } \\
\text { data }(n)\end{array}$ \\
\hline Mobility & $(0-28)$ & & & & & & & & & \\
\hline Overall sample & $2-28$ & $23.69(4.92)$ & $0 / 25.8$ & 0.85 & 0 & $2-28$ & $20.75(5.6 I)$ & $0 / 10.5$ & 0.86 & 0 \\
\hline EDSS 0-I.5 & $4-28$ & $25.08(3.62)$ & $0 / 34.2$ & 0.75 & 0 & $8-28$ & $24.22(3.65)$ & $0 / 21,7$ & 0.69 & 0 \\
\hline EDSS $2+$ & $2-28$ & $21.98(5.73)$ & $0 / 15.4$ & 0.88 & 0 & $2-28$ & $19.06(5.60)$ & $0 / 5.1$ & 0.85 & 0 \\
\hline Symptoms & $(0-28)$ & & & & & & & & & \\
\hline Overall sample & $3-28$ & $24.96(3.86)$ & $0 / 28.9$ & 0.82 & 0 & $0-28$ & $22.54(5.05)$ & $0.1 / 13.1$ & 0.85 & I \\
\hline EDSS 0-I.5 & $3-28$ & $26.01(2.86)$ & $0 / 37.8$ & 0.79 & 0 & $7-28$ & $24.81(3.50)$ & $0 / 25.2$ & 0.80 & 0 \\
\hline EDSS $2+$ & $7-28$ & $23.66(4.50)$ & $0 / 17.9$ & 0.82 & 0 & $0-28$ & $21.42(5.32)$ & $0.1 / 7.3$ & 0.85 & I \\
\hline Emotional Well-being & $(0-28)$ & & & & & & & & & \\
\hline Overall sample & $4-28$ & $24.14(4.32)$ & $0 / 23.8$ & 0.84 & 0 & $0-28$ & 23. II (4.96) & $0.1 / 17.4$ & 0.87 & 0 \\
\hline EDSS 0-I.5 & $10-28$ & $24.93(3.66)$ & $0 / 28.5$ & 0.80 & 0 & $4-28$ & $25.09(3.35)$ & $0 / 30.4$ & 0.79 & 0 \\
\hline EDSS 2+ & $4-28$ & $23.16(4.86)$ & $0 / 17.9$ & 0.86 & 0 & $0-28$ & $22.16(5.29)$ & $0.1 / I I .1$ & 0.88 & 0 \\
\hline General Contentment & $(0-28)$ & & & & & & & & & \\
\hline Overall sample & $3-28$ & $21.40(5.35)$ & $0 / 6.6$ & 0.86 & I & $2-28$ & $19.55(5.8 \mathrm{I})$ & $0 / 5.6$ & 0.85 & 0 \\
\hline EDSS 0-I.5 & $4-28$ & $22.44(4.81)$ & $0 / 8.8$ & 0.84 & 0 & $4-28$ & $22.48(4.50)$ & $0 / 9.6$ & 0.82 & 0 \\
\hline EDSS 2+ & $3-28$ & $20.11(5.71)$ & $0 / 3.9$ & 0.86 & I & $2-28$ & $18.14(5.84)$ & $0 / 3.6$ & 0.84 & 0 \\
\hline Thinking and Fatigue & $(0-36)$ & & & & & & & & & \\
\hline Overall sample & $5-36$ & $29.38(6.78)$ & $0 / 15.8$ & 0.93 & 0 & $0-36$ & $24.47(7.72)$ & $0.2 / 4.5$ & 0.90 & 0 \\
\hline EDSS 0-I.5 & $7-36$ & $31.28(5.03)$ & $0 / 20.7$ & 0.89 & 0 & $3-36$ & $28.46(6.22)$ & $0 / 9.5$ & 0.88 & 0 \\
\hline EDSS $2+$ & $5-36$ & $27.03(7.87)$ & $0 / 9.6$ & 0.94 & 0 & $0-36$ & $22.55(7.63)$ & $0.4 / 2.1$ & 0.89 & 0 \\
\hline Family/Social Well-being & $(0-28)$ & & & & & & & & & \\
\hline Overall sample & $5-28$ & $23.53(4.39)$ & $0 / 21.5$ & 0.78 & 0 & $0-28$ & $21.85(5.070$ & $0.1 / 14.0$ & 0.78 & I \\
\hline EDSS $0-1.5$ & $5-28$ & $24.23(4.22)$ & $0 / 26.9$ & 0.81 & 0 & $3-28$ & $23.69(4.23)$ & $0 / 23.4$ & 0.76 & 0 \\
\hline EDSS $2+$ & $6-28$ & $22.66(4.46)$ & $0 / 14.7$ & 0.75 & 0 & $0-28$ & $20.95(5.2 I)$ & $0.1 / 9.5$ & 0.77 & I \\
\hline Additional Concerns & $(0-56)$ & & & & & & & & & \\
\hline Overall sample & $20-56$ & $46.46(6.5 I)$ & $0 / 2.6$ & 0.78 & 0 & $12-56$ & $42.07(7.91)$ & $0 / 1.0$ & 0.78 & 20 \\
\hline EDSS 0-I.5 & $24-56$ & $47.79(5.84)$ & $0 / 4.1$ & 0.75 & 0 & $25-56$ & $46.29(6.01)$ & $0 / 2.3$ & 0.71 & 8 \\
\hline EDSS 2+ & $20-56$ & $44.82(6.93)$ & $0 / 0.6$ & 0.77 & 0 & $12-56$ & 40.05 (7.91) & $0 / 0.5$ & 0.76 & 12 \\
\hline
\end{tabular}

'symptoms' and 'thinking and fatigue' domains, and the TOI total (Table 4). The EDSS $2+$ subgroup demonstrated good responsiveness, with moderate to large standardized response means (SRMs) on almost all domains. Amongst the improved group all but one SRMs were lower for both EDSS subgroups.

The BEYOND EDSS 0-1.5 subgroup showed poor responsiveness amongst the worsened and improved groups, with the majority of domains and totals demonstrating small SRMs. Symptoms in the worsened group and 'thinking and fatigue' in the improved group, represented the highest levels of responsiveness, but these values were not representative of the subgroup. The EDSS $2+$ group showed higher effect sizes than the EDSS 0-1.5 group on all but one domain and totals in the worsened group. There was little difference in SRMs between the two EDSS groups in relation to those patients who had improved.
This appears contrary to the repeated measures t-test results, which are also presented for each trial subgroup in Table 4. The pattern of significance of the t-tests results suggest the FAMS sensitivity to improvement increases with baseline MS severity.

\section{Item response theory (IRT)}

IRT uni-dimensionality assumption. The 'additional concerns' domain of the FAMS includes items from all six of the other dimensions and so is not uni-dimensional. Thus, in order to be able to utilize these 14 items in the IRT analysis, an EFA with varimax rotation was conducted on all of the items in the FAMS. A six-factor solution was imposed on the data, in line with the number of domains that exist in the original FAMS, and the 'additional concerns' items were allocated to one of the six FAMS dimensions if their factor loading 
Table 3. The Functional Assessment of Multiple Sclerosis (FAMS) and Multiple Sclerosis Functional Composite (MSFC) construct validity correlations

\begin{tabular}{|c|c|c|c|c|c|c|c|c|c|c|c|c|}
\hline \multirow{3}{*}{ Correlation } & \multicolumn{6}{|c|}{ BENEFIT } & \multicolumn{6}{|c|}{ BEYOND } \\
\hline & \multicolumn{2}{|c|}{$\begin{array}{l}\text { Overall } \\
(n=348)\end{array}$} & \multicolumn{2}{|c|}{$\begin{array}{l}\text { EDSS } 0-1.5 \\
(n=193)\end{array}$} & \multicolumn{2}{|c|}{$\begin{array}{l}\text { EDSS } 2+ \\
(n=155)\end{array}$} & \multicolumn{2}{|c|}{$\begin{array}{l}\text { Overall } \\
(n=1634)\end{array}$} & \multicolumn{2}{|c|}{$\begin{array}{l}\text { EDSS 0-I.5 } \\
(n=535)\end{array}$} & \multicolumn{2}{|c|}{$\begin{array}{l}\text { EDSS } 2+ \\
(n=1099)\end{array}$} \\
\hline & $r$ & $p$-value & $r$ & $p$-value & $r$ & $p$-value & $r$ & $p$-value & $r$ & $p$ value & $r$ & $p$-value \\
\hline FAMS Total/MSFC Total & 0.31 & $<.01$ & 0.24 & $<.01$ & 0.29 & $<.01$ & 0.23 & $<.01$ & 0.20 & $<.01$ & 0.21 & $<.01$ \\
\hline FAMS TOI/MSFC Total & 0.28 & $<.01$ & 0.22 & $<.01$ & 0.23 & $<.01$ & 0.24 & $<.01$ & 0.22 & $<.01$ & 0.23 & $<.0 \mathrm{I}$ \\
\hline FAMS Mobility/MSFC Ambulation & 0.24 & $<.01$ & 0.15 & .04 & 0.32 & $<.01$ & 0.42 & $<.01$ & 0.17 & $<.01$ & 0.44 & $<.01$ \\
\hline FAMS Mobility/MSFC Arm Function & 0.21 & $<.01$ & 0.23 & $<.01$ & 0.09 & .28 & 0.43 & $<.01$ & 0.10 & .02 & 0.38 & $<.0 \mathrm{l}$ \\
\hline FAMS Thinking and Fatigue/MSFC Cognition & 0.16 & $<.01$ & 0.09 & .23 & 0.19 & .02 & 0.24 & $<.01$ & 0.12 & $<.01$ & 0.20 & $<.01$ \\
\hline
\end{tabular}

Table 4. Responsiveness analyses: repeated measures $t$-tests $(t)$ and standardized response means (SRMs)

\begin{tabular}{|c|c|c|c|c|c|c|c|c|c|c|c|c|c|c|c|c|}
\hline & \multicolumn{8}{|c|}{ BENEFIT } & \multicolumn{8}{|c|}{ BEYOND } \\
\hline & \multicolumn{4}{|c|}{ EDSS $0-1.5$} & \multicolumn{4}{|c|}{ EDSS $2+$} & \multicolumn{4}{|c|}{ EDSS $0-1.5$} & \multicolumn{4}{|l|}{ EDSS $2+$} \\
\hline & \multicolumn{2}{|c|}{$\begin{array}{l}\text { Worsened } \\
(n=53)\end{array}$} & \multicolumn{2}{|c|}{$\begin{array}{l}\text { Improved } \\
(n=29)\end{array}$} & \multicolumn{2}{|c|}{$\begin{array}{l}\text { Worsened } \\
(n=23)\end{array}$} & \multicolumn{2}{|c|}{$\begin{array}{l}\text { Improved } \\
(n=55)\end{array}$} & \multicolumn{2}{|c|}{$\begin{array}{l}\text { Worsened } \\
(n=126)\end{array}$} & \multicolumn{2}{|c|}{$\begin{array}{l}\text { Improved } \\
(n=4 I)\end{array}$} & \multicolumn{2}{|c|}{$\begin{array}{l}\text { Worsened } \\
(n=249)\end{array}$} & \multicolumn{2}{|c|}{$\begin{array}{l}\text { Improved } \\
(n=235)\end{array}$} \\
\hline & $\mathrm{t}$ & SRM & $\mathrm{t}$ & SRM & $\mathrm{t}$ & SRM & $\mathrm{t}$ & SRM & $\mathrm{t}$ & SRM & $\mathrm{t}$ & SRM & $\mathrm{t}$ & SRM & $\mathrm{t}$ & SRM \\
\hline Mobility & $2.55^{*}$ & 0.37 & 0.80 & 0.15 & $4.24 * * *$ & 0.88 & $2.24^{*}$ & 0.30 & $3.89 * * *$ & 0.35 & 1.84 & 0.29 & $10.09 * * *$ & 0.64 & $3.89 * * *$ & 0.25 \\
\hline Symptoms & $3.84 * * *$ & 0.55 & 1.32 & 0.25 & $5.26 * * *$ & 1.10 & 0.38 & 0.05 & $4.84 * * *$ & 0.43 & 1.01 & 0.30 & $4.80 * * *$ & 0.30 & $2.89 * *$ & 0.19 \\
\hline Emotional Well-being & 0.00 & 0.00 & 0.58 & 0.11 & $3.57 * *$ & 0.75 & $2.69 * *$ & 0.36 & $2.20 *$ & 0.20 & 0.63 & 0.10 & $7.86 * * *$ & 0.50 & 1.82 & 0.12 \\
\hline General Contentment & 1.08 & 0.17 & 0.43 & 0.08 & $3.44 * *$ & 0.72 & $3.36 * *$ & 0.45 & $2.00 *$ & 0.18 & 0.78 & 0.12 & $5.90 * * *$ & 0.37 & $4.21 * * *$ & 0.28 \\
\hline Thinking and Fatigue & $4.03 * * *$ & 0.56 & 0.98 & 0.18 & $2.16 *$ & 0.45 & 1.15 & 0.16 & 1.97 & 0.18 & $2.58^{*}$ & 0.40 & $3.80 * * *$ & 0.24 & $5.87^{* * *}$ & 0.38 \\
\hline Family/Social Well-being & 1.88 & 0.27 & 1.15 & 0.21 & 2.02 & 0.42 & 1.92 & 0.26 & 1.73 & 0.15 & 1.10 & 0.17 & $5.80 * * * *$ & 0.37 & 0.39 & 0.03 \\
\hline Additional Concerns & $2.98^{* * *}$ & 0.44 & 1.36 & 0.25 & $4.21 * * * *$ & 0.90 & 0.30 & 0.04 & $1.98^{*}$ & 0.18 & 0.91 & 0.04 & $8.41^{* * * *}$ & 0.54 & $3.05 * *$ & 0.20 \\
\hline Total & $2.95 * *$ & 0.42 & 1.01 & 0.19 & $4.60 * * *$ & 0.96 & $2.29 *$ & 0.31 & $3.32 * *$ & 0.30 & 1.05 & 0.16 & $8.89 * * * *$ & 0.54 & $4.42^{* * *}$ & 0.29 \\
\hline Trial Outcome Index & $3.90 * * *$ & 0.58 & 1.07 & 0.20 & $5.73 * * *$ & 1.20 & 1.30 & 0.18 & $2.80 * *$ & 0.25 & 1.61 & 0.25 & $8.59 * * * *$ & 0.54 & $4.44 * * *$ & 0.29 \\
\hline
\end{tabular}

Worsened $=$ EDSS progression $>0.5$ from baseline to end of study; Improved = EDSS reduction $>0.5$ from baseline to end of study. $* p$-value $\leq 0.05 ; * *$-value $<0.01$; $* * * p$-value $<0.001$.

was $\geq 0.4$, the cut-off value utilized in the FAMS development paper. ${ }^{5}$

One item loaded onto the general contentment domain and two items were shown to load onto each of the following domains: symptoms, emotional wellbeing domain, and family/social well-being. The remaining seven items did not sufficiently load onto any FAMS domain and were excluded.

Each of the six domains was then assessed for unidimensionality using EFA with a varimax rotation. In accordance with the Keiser Guttman criterion, ${ }^{24}$ domains were considered uni-dimensional if the first factor was the only factor to have an eigen value $>1$; items were retained for a dimension if their item loading was $\geq 0.4 .^{5}$ Two domains required some items to be removed or the domains restructured. The 'thinking and fatigue' domain was found to consist of two dimensions: fatigue and cognition. As such the IRT analysis was conducted on a fatigue domain (5 items) and a cognition domain (3 items); one item did not load sufficiently onto either domain and was excluded. In addition, two items were excluded from the family/social well-being domain due to insufficient factor loadings.

IRT calibration. Each domain was calibrated at baseline using a graded response model, ${ }^{23}$ the aim was to identify whether each domain could discriminate amongst individuals at higher levels of functioning by examining their test information curves (TICs). A high level of information suggests high discrimination or high reliability to discriminate. In accordance with the scoring of the FAMS, higher functioning is depicted by 


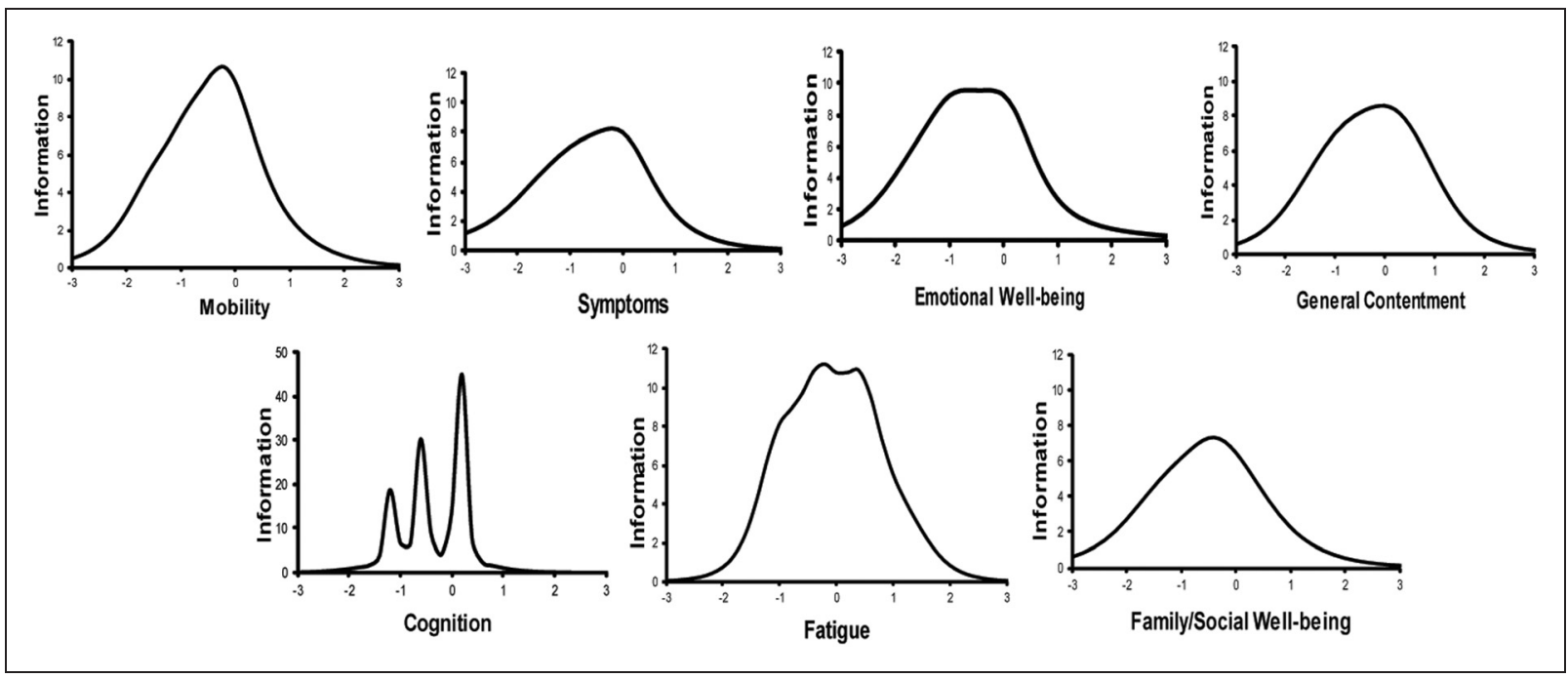

Figure I. Functional Assessment of Multiple Sclerosis domains test information curves: high levels of information at higher positive standard deviations reflect greater discrimination amongst the early multiple sclerosis population.

Table 5. Standardized measures of item response theory model precision

\begin{tabular}{|c|c|c|c|c|c|}
\hline \multirow{2}{*}{ Subscale } & \multicolumn{2}{|c|}{ Person location range } & \multicolumn{2}{|c|}{ Item difficulty range } & \multirow{2}{*}{$\frac{\text { Mean item difficult) }}{\text { Mean (SD) }}$} \\
\hline & Lower & Upper & Lower & Upper & \\
\hline General Contentment & -2.445 & 1.829 & -1.89 & 1.50 & $-0.332(0.98)$ \\
\hline Emotional Well-being & $-2.86 I$ & 1.735 & -2.46 & 2.27 & $-0.830(1.10)$ \\
\hline Family/Social Well-being & -2.502 & 1.451 & -1.820 & 0.743 & $-0.779(0.78)$ \\
\hline Fatigue & -1.827 & 1.759 & -1.077 & 1.235 & $-0.125(0.78)$ \\
\hline Mobility & -2.455 & 1.539 & -1.816 & 0.798 & $-0.655(0.82)$ \\
\hline Symptoms & -2.893 & 1.415 & -3.807 & 0.417 & $-1.287(1.07)$ \\
\hline Cognition & $-|.96|$ & 1.098 & -1.672 & 0.411 & $-0.645(0.7 I)$ \\
\hline
\end{tabular}

positive standard deviations in the standardized metric created for each latent trait (e.g. Mobility).

As illustrated in Figure 1, almost all domains failed to demonstrate the ability to discriminate amongst individuals with higher levels of functioning $(\mathrm{SD}>1)$. The fatigue and general contentment domains did demonstrate reasonable discrimination around $1 \mathrm{SD}$ above the mean, but this dropped dramatically by 2 SDs. The TIC information value can also be regarded as a measure of the reliability to discriminate, where reliability $=1-$ error variance or $1-(1$ /information $)$. As such, the TICs demonstrate low levels of reliability at 1 SD above the mean (0.5-0.6) for all domains except fatigue and general contentment which demonstrate good reliability $\sim 0.80$; however, in all instances reliability drops to zero at 2 SDs above the mean. Table 5 provides standardized indicators of the ranges of persons on a given dimension and associated item difficulties.

\section{Discussion}

This study assessed the psychometric properties of the FAMS within two distinct populations, CIS and early RRMS, with an aim to discern the appropriateness of the FAMS in trials involving these populations. Whilst the FAMS has been demonstrated as a valid and useful instrument for many years, there have been a number of changes relating to the treatment of MS, and the use of PROs, that postdate its development and require consideration. Recent years have seen a higher proportion of trials focusing on CIS and early treatment of MS. Treatments for MS such as IFN $\beta-1 b$ have been approved for use within CIS populations. In addition new guidance has emerged from regulators regarding the use of PROs. ${ }^{25,26}$ This indicates a need to reassess the measurement properties of the FAMS in people with suspected prodromal disease. This study provided one step towards this reassessment, with an evaluation 
of its psychometric properties amongst patients with CIS and early MS.

Traditional psychometric analysis of the CIS (BENEFIT) sample demonstrated good internal consistency on all domains for the sample as a whole and for each EDSS subgroup. These results, however, need to be considered in light of the high proportion of ceiling effects reported; these ceiling effects may over-inflate the levels of internal consistency. In addition, the ceiling effects indicate a lack of sensitivity or granularity of items at the higher level of functioning / lower level of impairment. This lack of granularity could also contribute to an explanation of the poor construct validity demonstrated by the FAMS in this population: in both EDSS subgroups the associations between MS functioning, as measured by the MSFC, and FAMS scores were small at best. However, the EDSS 2+ group did show higher correlations in almost all instances, suggesting that the FAMS construct validity improves amongst those with higher EDSS ratings, in line with the population used for its development.

The FAMS responsiveness results for the CIS population demonstrated some ability to detect change amongst those patients whose disease worsened. The EDSS $2+$ group showed greater effect sizes than those in the EDSS $0-1.5$ group, which may suggest an improvement in the FAMS ability to detect change when MS is more severe. However, neither EDSS subgroup demonstrated the FAMS ability to detect improvement. In the EDSS 0-1.5 group, even small effect sizes were rare, and whilst the EDSS 2+ group demonstrated higher effect sizes, no domain exceeded a small effect. The t-test results largely supported the SRM findings, with some evidence of significant improvement in four domains of the EDSS 2+ improvers group. This lack of sensitivity to detect improvement in the CIS population is likely to be explained by the high level of ceiling effects reported at baseline. However, this low sensitivity to change is also in line with a previous study assessing a more advanced MS population, ${ }^{6}$ which suggests this problem may extend beyond the CIS population.

The early MS (BEYOND) sample had a broader range of MS severity at baseline in the EDSS 2+ group than in the CIS sample (2-6.5 and 2-4, respectively), with higher median EDSS ratings (3 and 2, respectively). As suggested above, the traditional psychometric analyses, and the IRT analysis conducted on this early MS population, both suggested an improvement in the psychometric properties of the FAMS with increased disease/functioning severity.

The traditional psychometric analysis of the EDSS 0-1.5 group demonstrated good internal consistency, but as with the CIS population this was coupled with high ceiling effects, no evidence of construct validity and little evidence of responsiveness to improvement. However, unlike the CIS EDSS 0-1.5 group, the ability to detect decline in the early MS population was also poor, with only two domains demonstrating a small effect. This difference across populations could be due to the time difference in the trials and the baseline populations. The CIS trial (BENEFIT) was conducted over five years, permitting greater potential for decline from baseline to end of study compared to BEYOND, which was conducted over two years. Furthermore, the CIS populations decline over that five-year period could be more severe due to the potential for progression from CIS to clinically definite MS, which occurred for $49 \%$ of the sample.

The performance of the EDSS 2+ group amongst those with early MS, was superior to the $0-1.5$ group; in line with expectations that the FAMS performance would improve with MS severity. There was good internal consistency on all domains, and no domains demonstrated ceiling effects above the recommended maximum of $15 \% .{ }^{18}$ There was also some evidence of construct validity for the mobility domain, due to its correlation with the MSFC ambulation assessment. However, the natural restriction in range of FAMS and MSFC scores amongst the EDDS 0-1.5 group compared to the EDSS $2+$ group (with an EDSS range of 2-6.5) may also contribute to the differences in correlations between the subgroups.

The responsiveness analysis also demonstrated small to moderate effect sizes for detecting EDSS progression. Again, the effect sizes seen in BEYOND were not as high as those recorded in BENEFIT; however, as above, this can be explained in part by the trial length and study sample. The fact that both trials demonstrate a general pattern of increased effect size from the $0-1.5$ groups to the $2+$ groups supports the idea of an improvement in the FAMS psychometric properties with increased disease severity. However, even the early MS population's EDSS 2+ group did not demonstrate sensitivity to change amongst patients who improved from baseline to end of study, according to the SRM findings. The $t$-test results appear to suggest otherwise, with highly significant changes reported in the EDSS $2+$ improvers group. However, the sample size differences between the EDSS $0-1.5$ and $2+$ subgroups are likely to be responsible for this pattern of results rather than the actual magnitude of change, which is better captured by the SRM values. Again, these results support previous reports of the FAMS low sensitivity to change, even amongst more advanced MS populations. ${ }^{6}$ Given that $17 \%$ of the BEYOND population demonstrated improvement, an improved ability to detect change is necessary.

The IRT analysis supported the other findings. A reduced level of reliability was demonstrated at higher 
levels of functioning with only two domains, demonstrating sensitivity or reliability at $1 \mathrm{SD}$ above the mean. There was no evidence of sensitivity or reliability in any domain at 2 SDs above the mean. The measures of model precision provide further support, suggesting greater sensitivity of the instrument at the more severe end of domain spectrums. As suggested by the traditional psychometric results, the IRT analysis highlights the need for greater sensitivity of items at higher levels of functioning.

\section{Limitations}

Owing to the MS populations under consideration and the post-hoc nature of this analysis, there are some limitations to be considered. Arguably, the method used to assess sensitivity to change in the current study was limited; the post-hoc nature of the analysis prevented the employment of a more robust method, such as a patientbased anchor method. While a 0.5 change in EDSS was selected based in clinician input, the impact of a 0.5 change on a patient is not uniform across the EDSS scale, and applying this criterion to those with low EDSS scores may limit the extent to which the FAMS scores would be expected to change. However, as previous assessments of the FAMS sensitivity to change found similar results using a patient-based anchor approach ${ }^{6}$ this study's limitations are unlikely to fully account for the FAMS low sensitivity to change results.

Furthermore, as alluded to above, when examining the assessment of construct validity, consideration must be given to the fact that a natural limitation of focusing on particular strata of MS severity is that it imposes a restriction in the range of scores. As such, this restriction may in part account for the low correlations in both samples overall and the higher correlations in the EDSS 2+ groups compared to the EDSS 0-1.5 groups.

\section{Conclusions}

The FAMS may remain a valid and useful instrument in moderate to severe MS; however, given the increased emphasis on trials investigating early treatment in MS and CIS populations, it is imperative that the instruments utilized in such trials are psychometrically valid. Both the CIS and early MS samples included in these analyses support the improvement of the FAMS psychometric properties with increased baseline disease severity. However, the high ceiling effects at low EDSS levels coupled with the persistent lack of responsiveness to improvement and poor construct validity suggest that the FAMS is not functioning amongst those with less severe MS. Based on these results and recent guidelines on PRO development and use, ${ }^{26}$ it seems that further qualitative research to establish the content validity of the FAMS in CIS and early MS populations is required before any further statistical psychometric evaluations are conducted. This work to establish the relevance of the FAMS concepts/items and make any required modifications is imperative if the FAMS is ever to be used as a clinical trial endpoint in these populations.

\section{Funding}

This research was funded by Bayer HealthCare Pharmaceuticals.

\section{Conflict of interest statement}

C. Wang, V. Knappertz and K. Gondek are employees of Bayer HealthCare Pharmaceuticals; B. Stemper, K Beckmann, C. Pohl and R. Sandbrink are employees of Bayer Schering Pharma.

\section{References}

1. Scoggins JF and Patrick DL. The use of patient-reported outcome instruments in registered clinical trials: evidence from ClinicalTrials.gov. Contemp Clin Trials 2009; 30: 289-292.

2. Solari A. Role of health-related quality of life measures in the routine care of people with multiple sclerosis. Health Qual Life Outcomes 2005; 3: 16.

3. Kurtzke JF. Rating neurologic impairment in multiple sclerosis: an expanded disability status scale (EDSS). Neurology 1983; 33: 1444-1452.

4. Nortvedt MW, Riise T, Myhr KM and Nyland HI. Quality of life in multiple sclerosis: measuring the disease effects more broadly. Neurology 1999; 53: 1098-1103.

5. Cella DF, Dineen K, Arnason B, Reder A, Webster KA, Karabatsos G, et al. Validation of the Functional Assessment of Multiple Sclerosis quality of life instrument. Neurology 1996; 47: 129-139.

6. Giordano A, Pucci E, Naldi P, Mendozzi L, Milanese C, Tronci F, et al. Responsiveness of patient reported outcome measures in multiple sclerosis relapses: the REMS study. J Neurol Neurosurg Psychiatry 2009; 80: 1023-1028.

7. Hobart JC, Riazi A, Lamping DL, Fitzpatrick R and Thompson AJ. How responsive is the Multiple Sclerosis Impact Scale (MSIS - 29)? A comparison with some other self-report scales. J Neurol Neurosurg Psychiatry 2005; 76: 1539-1543.

8. Riazi A, Hobart JC, Lamping DL, Fitzpatrick R and Thompson AJ. Evidence based measurement in multiple sclerosis: the psychometric properties of the physical and psychological dimensions of three quality of life rating scales. Mult Scler 2003; 9: 411-419.

9. European Medicines Agency. Committee for Medicinal Products for Human Use: Draft Guideline on Clinical Investigation of Medicinal Products for the Treatment of multiple Sclerosis. 2005. Available at http://www. ema.europa.eu/pdfs/human/ewp/056198en.pdf. Accessed 21 October 2010. 
10. European Medicines Agency. Committee for Medicinal Products for Human Use: Guideline on Clinical Investigation of Medicinal Products for the Treatment of multiple Sclerosis. 2006. Available at http://www. ema.europa.eu/pdfs/human/ewp/56198en.pdf. Accessed 21 October 2010.

11. Kappos L, Polman CH, Freedman MS, Edan G, Hartung HP, Miller DH, et al. Treatment with interferon beta-1b delays conversion to clinically definite and McDonald MS in patients with clinically isolated syndromes. Neurology 2006; 67: 1242-1249.

12. Kappos L, Freedman MS, Polman CH, Edan G, Hartung HP, Miller D, et al. Effect of early versus delayed interferon beta-1b treatment on disability after a first clinical event suggestive of multiple sclerosis: a 3-year follow-up analysis of the BENEFIT study. Lancet 2007; 370: 389-397.

13. Kappos L, Freedman MS, Polman CH, Edan G, Hartung HP, Miller D, et al. Long term effect of early treatment with interferon beta- $1 \mathrm{~b}$ after a first clinical event suggestive of multiple sclerosis: a 5-year active treatment extension of the phase 3 BENEFIT trial. Lancet Neurol 2009; 8: 987-997.

14. O'Connor P, Filippi M, Arnason B, Comi G, Cook S, Goodin D, et al. Interferon beta $1-b \quad 250 \mathrm{mg}$ versus interferon beta $1-b \quad 500 \mathrm{mg}$ versus glatiramer acetate in relapsing-remitting multiple sclerosis: results of a prospective, randomised multicentre study. Lancet Neurol 2009; 8: 889-897.

15. Edelen M and Reeve B. Applying item response theory (IRT) modeling to questionnaire development, evaluation and refinement. Qual Life Res 2007; 16: 5-18.

16. Fries JF, Bruce B and Cella D. The promise of PROMIS: using item response theory to improve assessment of patient reported outcomes. Clin Exp Rheumatol 2005; 23(Suppl. 39): S53-S57.
17. Ruddick RA, Cutter G and Reingold S. The Multiple Sclerosis Functional Composite: a new clinical outcome measure for multiple sclerosis trials. Mult Scler 2002; 8: 359-365.

18. McHorney CA and Tarlov AR. Individual patient monitoring in clinical practice: are available health status surveys adequate? Qual Life Res 1995; 4: 293-307.

19. Nunnally JC and Bernstein IH. Psychometric Theory, 3rd ed. New York: McGraw-Hill Series in Psychology, 1994.

20. Kline P. The Handbook of Psychological Testing, 2nd ed. London: Routledge, 2000.

21. Stucki G, Liang MH, Fossel AH and Katz JN. Relative responsiveness of condition-specific and generic health status measures in degenerative lumbar spinal stenosis. J Clin Epidemiol 1995; 48: 1369-1378.

22. Zou GY. Quantifying responsiveness of quality if life measures without an external criterion. Qual Life Res 2005; 14: 1545-1552.

23. Samejima F. Estimation of latent ability using a response pattern of graded scores. Psychometrika Monograph Supplement 1969, No. 17.

24. Kaiser HF. The application of electronic computers to factor analysis. Educ Psychol Meas 1960; 20: 141-151.

25. Food and Drug Administration. Guidance for Industry. Patient-reported outcome measures: Use in medical product development to support labeling claims. Draft Guidance. US Department of Health and Human Services, 2006. Available at http://www.fda.gov/cder/ guidance/5460dft.pdf. Accessed 21 October 2010.

26. Food and Drug Administration. Guidance for Industry. Patient-reported outcome measures: Use in medical product development to support labeling claims. Available at US Department of Health and Human Services. 2009. http://www.fda.gov/downloads/Drugs/GuidanceComplia nceRegulatoryInformation/Guidances/UCM193282.pdf. Accessed 21 October 2010. 